Meta

Journal des tradlucteurs

Translators' Journal

\title{
Bibliographie de la traduction médicale
}

\section{Henri Van Hoof}

Volume 19, numéro 1, mars 1974

La traduction médicale

URI : https://id.erudit.org/iderudit/003090ar

DOI : https://doi.org/10.7202/003090ar

Aller au sommaire du numéro

\section{Éditeur(s)}

Les Presses de l'Université de Montréal

\section{ISSN}

0026-0452 (imprimé)

1492-1421 (numérique)

Découvrir la revue

Citer ce document

Van Hoof, H. (1974). Bibliographie de la traduction médicale. Meta, 19(1), 43-66. https://doi.org/10.7202/003090ar d'utilisation que vous pouvez consulter en ligne.

https://apropos.erudit.org/fr/usagers/politique-dutilisation/ 


\section{Bibliographie de la traduction médicale}

\section{BIBLIOGRAPHIE DE LA BIBLIOGRAPHIE}

XXX, Verzeichnis von Wörtenbüchern über Medizin (1950-1963), Berlin, ZentralInstitut für Information u. Dokumentation, 1963, MB no 106, $5 \mathrm{p}$.

Chatton et Sanazaro, Current Medical References, Lange, 1967, 595 p.

Cunningham, Classification for Medical Literature, Vanderbilt University, 1967, $267 \mathrm{p}$.

Blake et Roos, Medical Reference Works (1679-1966), Chicago (Ill.), Medical Library Association, 1967.

Bottle et Wyatt, The Use of Biological Literature, Archon, Shoe String Press, 1967, $286 \mathrm{p}$.

Brodman, Development of Medical Bibliography, Chicogo (Ill.), Medical Library Association, 1954, $226 \mathrm{p}$.

Kricker, G., Die Schrifttumnachweise der Medizin, 1944.

Saur, K. et G. Gringmuth, Wörterbücher und Lexika - Medizin, Biologie, Gesundheitswesen, Pharmazie, Veterinärmedizin, Munich, Verlag Dokumentation - Pullack, 1969, $34 \mathrm{p}$.

\section{ARTICLES}

Anzlowar, B.R., «Biomedical Translations in the Coming Decade», ATA Notes (New York), mars 1965.

Bentz, H.W., "Sigmund Freud in Übersetzungen », Reihe (Francfort), 1, Bd2, $1961,60 \mathrm{p}$.

Boerman, A.J., «Bedside Manners » (trad. médicale), Van Taal tot Taal, vol. 1, $\mathrm{n}^{\circ} 1,1956$, p. $10-11$.

Boulay, J. $\left(\mathrm{D}^{\mathrm{r}}\right)$, «Traduction médicale », Meta, vol. 13, $\mathrm{n}^{\circ}$ 3, 1968, p. 127-132. 
Chartrand, L., "Quelques problèmes de traduction de pharmacie », Journal des traducteurs, vol. 2, $\mathrm{n}^{\circ} 1,1957$, p. 16-19.

Du Jardin, J.L., «Gelber's The Connection, Problems of Translation DrugAddiction in French and English », Mémoire (Anvers), 1967, 149 p.

Fischbach, H., «Problems of Medical Translation », Bulletin of Medical Library Association, vol. 50, $\mathrm{n}^{\circ} 3,1962$, p. 462-472.

Fischbach, H., «What the Translation Client Should Know » (traduction médicale ), Journal des traducteurs, vol. 6, $\mathrm{n}^{\circ} 4,1961$, p. 111-121.

Flöttmann, W., «Translation of Scientific Papers : Materia Medica, Lebende Sprachen, vol. 12, $\mathrm{n}^{\circ}$ 1, 1967, p. 4-5.

Laplancke, J. et J.-F. Pontalis, «Connaître Freud avant de le traduire », le Monde (Paris), $1^{\text {er }}$ mars 1967.

Limbos, M., "Terminologie van de lepra (avec lexique Ne-Fr/F-Ne sur la lèpre) 》, Mémoire (Anvers), 1963, $312 \mathrm{p}$.

Lord, R., « Traduction et pharmacie : évolution du vocabulaire de la pharmacie », Journal des traducteurs, vol. $2, \mathrm{n}^{\circ} 1,1957, \mathrm{p} .10-12$.

Mary, A., "Le traducteur dans l'industrie », compte rendu d'une conférence sur la traduction pharmaceutique par M. Schnarrenberger, l'Interprète, vol. 23, $\mathrm{n}^{\circ} 3,1968$, p. 10-13 ; Traduire, $\mathrm{n}^{\circ} 60,1969$, p. 8-11.

Mayrand, A., «La traduction en pharmacie : le devoir d'information du pharmacien et l'aspect juridique du bilinguisme $»$, Journal des traducteurs, vol. 2, $\mathrm{n}^{0} 1,1957$, p. 7-9.

Monnerot-Dumaine $\left(\mathrm{D}^{\mathrm{r}}\right)$, « Terminologie et lexicographie médicales », Presse médicale (Paris), vol. 75, $\mathrm{n}^{\circ} 19,1967$, p. 996-997.

Sliosberg, A., "Quelques considérations sur la traduction médicale et pharmaceutique $»$, communication au $29^{\mathrm{e}}$ Congrès international de sciences pharmaceutiques de la Fédération internationale pharmaceutique, Londres, septembre 1969, Traduire, $\mathrm{n}^{\circ} 63,1970$, p. 15.

Sliosberg, A., "Quelques considérations sur la traduction médicale et pharmaceutique , le Linguiste, vol. 15, $\mathrm{n}^{\circ} 6,1969$, p. 1-6 ; Babel, vol. 17, $\mathrm{n}^{\circ} 1,1971$, p. 14-21.

Vacariu, G., « Réflexions sur la traduction médicale », Journal des traducteurs, vol. $2, \mathrm{n}^{\circ} 1,1957$, p. 13-15.

Van Hoof, $\mathrm{H}$., «La traduction médico-pharmaceutique », le Linguiste, vol. 5, $\mathrm{n}^{\circ} 1$, 1959 , p. $8-12 ; \mathrm{n}^{\circ} 2$, p. $5-8 ; \mathrm{n}^{\circ} 4$, p. $6-9$; Lebende Sprachen, $\mathrm{n}^{\circ} 5,1960$, p. 43-76, 115-171.

Van Hoof, $\mathrm{H}$., «La traduction médico-pharmaceutique », communication au Colloque sur le langage médical, Liège, octobre 1969, le Langage et l'homme, vol. $4, \mathrm{n}^{\circ} 12,1970$, p. 65-77 ; Meta, vol. 15, n 2, p. 95-109.

Van Hoof, H., «Réflexions sur le langage médical : le point de vue du traducteur scientifique $»$, la Presse médicale (Paris), vol. 67, $\mathrm{n}^{\circ} 11,1959$, p. 419-421. 


\section{MANUELS}

\subsection{DOMAINE FRANÇAIS}

3.1.1. Ouvrages unilingues français

Conseil des Organisations internationales des sciences, Terminologie et lexicographie médicales, C.v. des travaux d'un groupe international d'experts, Paris, Masson et $\mathrm{C}^{\mathrm{te}}, 1967,60 \mathrm{p}$.

\subsection{DOMAINE ANGLAIS}

\subsubsection{Ouvrages unilingues anglais}

Allan, F.D. et J.B. Christensen, The Language of Medecine, Washington (D.C.), Sigma Press, 1966, 96 p.

Close, P.A., The English we Use for Science (onze textes médicaux; intéressante introduction), Londres, Longmans, 1968, 210 p.

Field, D.J. et J.B. Harrison, Anatomical Terms, their Origin and Derivation, $3^{e}$ éd., Cambridge, Heffer, 1968, 212 p.

Martins, J.M., Englìsh for the Foreign Physician, $4^{\mathrm{e}}$ éd., Springfield (H1.), Thomas, $1968,136 \mathrm{p}$.

Morton, L.T., How to Use a Medical Library - A Guide for Practitioners, Research Workers and Students, Londres, Heinemann, 1964, 72 p.

Roberts, R., Medical Terms, their Origin and Construction, $4^{\mathrm{e}}$ éd., Londres, Heinemann, 1966, 96 p.

Schmidt, J.E., Structural Units of Medical and Biological Terms, Springfield (IIl), Thomas, $1969,156 \mathrm{p}$.

Skinner, H.A., The Origin of Medical Terms, $2^{\circ}$ éd., Baltimore, Williams and Wilkins, 1961, $438 \mathrm{p}$.

3.2.2. Ouvrages à partir d'autres langues

Kupferschmidt, H.G., English für Mediziner (avec lexique En-De), $4^{\ominus}$ éd., Leipzig (RDA), Verlag Enzyklopädie, 1967, 320 p.

\section{DICTIONNAIRES, LEXIQUES, TERMINOLOGIES}

\subsection{MÉDECINE EN GÉNÉRAL}

Dictionnaire médical anglo-russe, Paris, Éditions de Moscou, 1958. [20,90 F]

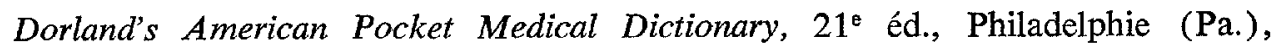
Saunders, 1968 , ill. 715 p. [\$5.75]

Encyclopédie médicale pour tous, Bruxelles, Sequoia, 1961, ill., 220 p. [F.B. 85,-] Encyclopédie médicale pratique, Paris, Denoël, 1966, ill., 544 p. [39 FF]

Nomenclature générale des actes professionnels des médecins, chirurgiens, dentistes, sages-femmes et auxiliaires médicaux, Paris, Librairies techniques, 1960 , publication annuelle. $[8,-\mathrm{FF}]$

Comité Clair-Dire, «Table d'anglicismes médicaux et leurs équivalents», le Linguiste (Bruxelles), 1965, $\mathrm{n}^{\circ} 4-5$, p. 11-14. 
US' National Institutes of Health, Medical and Health Related Sciences Thesaurus, 1963.

Abercrombie, G. F. et R.M.S. Mc Conaghey, The Encyclopaedia of General Practice, 7 vol., Londres, Butterworth, 1967, ill., 3600 p. [£35/-]

Agnew, L.R.C. et al., Dorland's Illustrated Medical Dictionary, 24e éd., Philadelphie, W.B. Saunders, 1965, ill., $19+1724$ p. [\$13.00]

Aoyagi, A. et al., Medical Terminology, Latin, French, English, German, Tokyo, 1958,610 p. [\$10.00]

Bonvalot, M. et al., le Vocabulaire médical de base, 4 vol., Paris, O.I.P., 1969, 480 p. $[60,-\mathrm{FF}]$

Brown, J.A.C., Pears Medical Encyclopaedia, Londres, Pelham Books, 1971, ill., 464 p. $[£ 3,50]$

Brussel, J.A. et al., Medical Aid Encyclopaedia for the Home, New York, Stravon Educ. Pr., 1965, $8+648$ p. [\$14.50]

Clairville, A.L., Dictionnaire polyglotte des termes médicaux, $2^{\circ}$ éd., Paris, SIPUCO, 1953, 1152 p. [65,-FF]

Clare, Understanding Medical Terminology, $3^{\mathrm{e}}$ éd., Catholic Hospital Assoc., 1966,246 p. [\$5.00]

Cohen of Birkenhead, Lord, British Encyclopaedia of Medical Practice Medical Progress, Londres, Butterworth, 1967, 456 p. [£3/12/—]

Dabout, E. et Neveu, Petit Dictionnaire de médecine, $2^{2}$ éd., Paris, Baillière, 1949, 738 p. [DM 15,80]

Davies, P.M., Medical Terminology in Hospital Practice, Londres, Heinemann, 1969,344 p. [30/-sh]

Del Guercio, L.R.M. et al., The Multilingual Manual for Medical Interpreting (English-French-Spanish-Italian-Russian), $2^{\circledR}$ éd., New York, Pacific Printing, 1960,160 p.

Edmonson, F.W., Medical Terminology, 4e éd., New York, Putnam, 1965, $14+$ 306 p. [\$4.95]

Fishbein, A.M., Modern Woman's Medical Encyclopaedia, New York, Avon, 1968,508 p. [\$1.25]

Galtier-Boissière et al., Nouveau Larousse médical illustré, Paris, Larousse, 1955, ill., 1248 p. $[68,20 \mathrm{FF}]$

Garnier, M. et V. Delamare, Dictionnaire des termes techniques de médecine, $18^{e}$ éd., Paris, Maloine, 1967, $8+1301$ p. [19,-FF]

Gaucher, M. et al., Encyclopédie médicale Quillet : nouvelle encyclopédie pratique de médecine et d'hygiène, Paris, Quillet, 1965, ill., $16+8+883$ p. $[120,-$ FF $]$

Gladstone, W.J., Vocabulaire de médecine et des sciences connexes, Paris, Masson, 1971,295 p. [48,-FF]

Gomez, J., A Dictionary of Symptoms : A Medical Dictionary to Help Sufferers, New York, Stein \& Day, 1968, ill., 312 p. 
Gordon, B.L., et al., American Medical Association, Current Medical Terminology, $3^{e}$ éd., Chicago (Ill.), AMA, 1966, 969 p.

Gould, G.M., C.V. Brownlow, et al., Gould's Pocket Pronouncing Medical Dictionary, $11^{\mathrm{e}}$ éd., New York, Blakiston, 1947.

Goust, F., la Médecine pour tous (avec index alphabétique), Paris, Larousse, $1954,812 \mathrm{p}$.

Hamburger, J., Petite encyclopédie médicale, 13e éd., Paris, Flammarion, 1968, ill., 1224 p. $[65,-$ FF $]$

Hermans, A.G., Vademecum Medicum Polyglottum, Stockolm, Den Haag, 1946, $62 \mathrm{p}$.

Hill, Ch., The Radio Doctor's Dictionary of Health, Londres, Dent, 1963, 304 p.

Hirsch, C.J. et J.A. Curtin, Medecine, 3 vol., New York, Macmillan, 1966, ill., $85+83+43 \mathrm{p}$.

Hoerr, N.L. et al., Blakiston's Illustrated Pocket Medical Dictionary, $2^{\mathrm{e}}$ éd., New York, McGraw-Hill, 1960, ill., $12+983$ p.

Hoerr, N.L., A. Osol et al., Blakiston's New Gould Medical Dictionary, $2^{e}$ éd., New York, McGraw-Hill, 1957, ill., 26 + 1462 p.

Horder, Lord, British Encyclopaedia of Medical Practice (including medecine, surgery, obstetrics, gynaecology and other special subjects), Londres, Butterworth, 1955-1968, 12 vol. + pharmacopée + index + 6 suppl., 8000 p. + 5300 p. [£45/10+£21/10]

Hyman, H. Th., The Complete Home Medical Encyclopaedia, éd. augm., New York, Avon, 1965 , ill., 832 p. [\$1.25]

Iwanami, Medical Terminology, Tokyo, 1958, 615 p. [DM 45,-]

Jaeger, E.C., A Source-Book of Medical Terms, Springfield (I11.), Thomas, 1953, ill., 170 p. [\$5.50]

Lepine, P. et G.D. Krasnoff, Dictionnaire français-anglais, anglais-français des termes médicaux et biologiques, Paris, Éd. médicales Flammarion ; Londres, Lewis, 1952, $291+538$ p.

MacNalty, The British Medical Dictionary (and Supplement), 1961-1962.

MacNalty, A.S., Butterworth's Medical Dictionary (anct. : The British Med. Dict.) - Supp. 1965, Londres, Butterworth, 1965, 1717 p. [147/6 sh]

Manuila, A., L. Manuila, M. Nicole et $\mathrm{H}$. Lambert, Dictionnaire français de médecine et de biologie, 4 vol., Paris, Masson, vol. parus : 1970, t. I, $A-D, 49+$ 816 p. ; 1971, t. II, $E-M, 923$ p.

McCullogh, J.A., A Medical Greek and Latin Wordbook, Springfield, Thomas, 1962,168 p. [\$5.75]

Nicole, G. et M., Classification médicale de la National Library of Medecine, traduction de l'original américain, Paris, Gauthier-Villars, 1970, 312 p.

Oakes, L., Pocket Medical Dictionary, Edimbourg, E. \& S. Livingstone, 1961. [8/6 sh.]

Paddock, M.J., Basic Medical Terms and Techniques Simplified, Chicago, Am. Technol. Society, 1955, $147 \mathrm{p}$. 
Parr, J.A. et R.A. Young, Concise Medical Encyclopaedia, Amsterdam, Elsevier, $1965,12+514$ p. [fl. 20,00]

Parry, S., Polyglot Medical Questionnaire in Twelve Languages, Londres, Lewis, 1953, 62 p. [DM 9,35]

Pearce, E.C., Medical and Nursing Dictionary and Encyclopaedia, $13^{\mathrm{e}}$ éd., Londres, Faber \& Faber, 1966, $3+578$ p. [50/-sh.]

Ridayre, B. et al., Dictionnaire Marabout de la médecine, 7 vol., Verviers (Belgique), Gérard, 1967-1969, ill., format de poche, $286+285+282+284+$ $284+236+222 \mathrm{p}$.

Riley, P.A. et P.J. Cunningham, The Faber Pocket Medical Dictionary, Londres, Faber \& Faber, 1966. [9/6 sh.]

Roger, N., Livingstone's Pocket Medical Dictionary, $11^{\circ}$ éd., Édimbourg-Londres, Livingstone, 1969, ill., 616 p. [9/6 sh.]

Rothenberg, R.E., The New American Medical Dictionary and Health Manual, New York, New Amer. Libr., 1968, ill., 496 p.

Schmidt, J.E., Dictionary of Medical Slang and Related Esoteric Expressions, Springfield (Ill.), Thomas, Oxford, Blackwell Scient. Publ., 1959, 224 p. [32/-sh.]

Schmidt, J.E., Paramedical Dictionary, Springfield (IIl.), Thomas, 1969, 423 p. [\$8.75]

Schmidt, J.E., Revision : A Medical Word Finder, Springfield (Ill.), Thomas, 1958,456 p. [\$7.50]

Sichulz, H.E., Vocabularium Medicinale Polyglotte, 2 vol., Halle (Westf.), Deutsches Nomenklatur-Arch., 1963-1964, 270 p. [DM 65,-]

Sliosberg, A., Encyclopédie médicale, 2 vol., Paris, Nathan, 1966, ill., 908 p. [le vol., 80,- - FF]

Sliosberg, A., Medical Dictionary, English-German-French-Spanish-Italian, Amsterdam, Elsevier, 1964, $8+1588$ p. [fl. 125,-]

Smith, G.L. et P.E. Davis, Medical Terminology : A Programmed Text, $2^{\mathrm{e}}$ éd., New York, J. Wiley, 1967, ill., 289 p. [\$5.50]

Spilman, M., Medical Latin and Greek, $2^{\mathrm{e}}$ éd., Salt Lake City (Ut.), 1949, 39 p.

Stanton, I.A., A Dictionary for Medical Secretaries, Springfield (I11.), Thomas, 1960,184 p. [\$6.50]

Strand, H.R., An Illustrated Guide to Medical Terminology, Baltimore (Md.), Williams \& Wilkins, 1968 , ill., 110 p. [\$5.75]

Taber, C.W. et al., Taber's Cyclopaedic Medical Dictionary, $10^{\circ}$ éd., Oxford, Blackwell Scientif. Publ., 1965, ill., 1408 p. [£2/6/-]

Taylor, N.B., A.E. Taylor et al., Stedman's Medical Dictionary, $21^{\mathrm{e}}$ éd., Baltimore, Williams \& Wilkins, 1967, ill., 1836 p. [\$14.00]

Taylor, N.B. et A.E. Taylor, The Putnam Medical Dictionary, New York, Putnam, 1961,933 p. [\$4.95]

Thomson, W.A.R. et al., Black's Medical Dictionary, $28^{\circ}$ éd., Londres, A. \& C. Black, 1968, ill., 1012 p. [42/—sh.] 
Tsinikas, T. et al., Encyclopédie médicale moderne et illustrée, 6 vol., Athènes, 1960 , ill., 2750 p. [DM 160,-]

Veilion, E. et A. Nobel, Dictionnaire médical - Mediziniscees Wötterbuch Medical Dictionary, 5e éd., Bern, Hans Huber, 1969, $22+1329$ p. [DM 138,-]

Wakeley, C., Sir, The Faber Medical Dictionary (with a 1965 Supplement), Londres, Faber \& Faber, 1953-1965, 7 + 471 p. + suppl.

Willeford, G., Medical Word Finder, West Nyack (N.Y.), Parker, 1967, 340 p. [\$6.95]

Young et Barger, Learning Medical Terminology Step by Step, St. Louis (Minn.), Mosby, 1967, 341 p. [\$7.50]

\subsection{DISCIPLINES PARTICULIÈRES}

4.2.1. Abréviations, symboles

Michigan Occupational Therapy Association, Medical Abbreviations : A Cross Reference Dictionary, $2^{\mathrm{e}}$ éd., Ann Arbor (Mich.), Univ. Hospital, Dept. of Physical Med. and Rehabilitation, 1967, $7+165$ p. [\$3.00]

World Health Organization, Abbreviations for Hormones and Related Substances (English, French, Spanish), TC/3, 1964, 7 p.

Hiebert, Abbreviations of Basic Medical Physiology, $4^{\circ}$ éd., New York, Sigma Press, 1962,320 p. [\$7.50]

Kerr, A.H., Medical Hyeroglyphs, Abbreviations and Symbols, Chicago, 383 p. [\$17.50]

Lerebouillet, J. et al., Abréviations utilisées en médecine et en biologie médicale (anglais-allemand-espagnol-français-italien-portugais), Paris, Union internationale de la presse médicale, $1965,96 \mathrm{p}$. [15,50 FF $]$

Peyser, A., Pars Pro Toto : Breviarum Medicum international, anglais-allemandespagnol-français-italien (abréviations dans la litt. intern. de la médecine et des sciences annexes), Stockholm, Almquist \& Wiksell, 1950, 196 p. [DM $10,75]$

Steen, E.B., Dictionary of Abbreviations in Medicine and the Related Sciences, $2^{\mathrm{e}}$ éd., Philadelphie, F.A. Davis, 1963, 102 p. [\$2.50]

Touati, M., Lexique des abréviations et formules médico-chirurgicales, $2^{\mathrm{e}}$ éd., Paris, Maloine, 1968, 142 p. [20,- FF]

\subsubsection{Allergologie}

Sabouraud, «Lexique des principaux termes utilisés en allergologie », Gaz. méd. France, 1968, vol. 5, p. 4089-4095.

Wilken-Jensen, K., Lexicon allergologicum, $2^{\circ}$ éd., Londres, Pergamon Press, 1966, 120 p. $[\$ 5.00]$

4.2.3. Anatomie

International Anatomical Nomenclature Committee, Nomina Anatomica, 1950, $83 \mathrm{p}$. 
Anson, B.J., Atlas of Human Anatomy, $2^{\text {e }}$ éd., Philadelphie, Saunders, 1963, ill., 632 p. [\$18.00]

Antunez, L., Atlas of Human Anatomy, Londres, Blackwell, 1969, 350 p. [62/6 sh. $]$

Bossy, J., Atlas of Neuroanatomy, Londres, Blackwell, 1969, ill., 375 p. [70/- sh.]

Dickinson, Atlas of Human Sex Anatomy, 2e éd., Baltimore, Williams \& Wilkins, $1949,346 \mathrm{p}$.

Dobson, J., Anatomical Eponyms - A Biographical Dictionary of those Anatomists whose Names have become Incorporated into Anatomical Nomenclature, $2^{2}$ éd., Édimbourg, Livingstone, 1962, $9+240$ p. [30/- sh.]

Donath, T., Anatomical Dictionary with Nomenclature and Explanatory Notes, Londres, Pergamon Press, 1969, 606 p.

Ford, D.H. et J.P. Schadé, Atlas of the Human Brain, Amsterdam, Elsevier, 1966, réimp. 1967, ill., $7+166$ p. [fl. 30,-]

Frohse, F. et al., Atlas of Human Anatomy - College Outlines, New York, Barnes \& Nobles, 1961, $180 \mathrm{p}$.

Grant, J.C.B., Atlas of Anatomy, 5e éd., Londres, Blackwell, 1962.

Lovasy, E. et E. Veillon, Dictionnaire des termes d'anatomie, d'embryologie et d'histologie, Paris, 1954, 624 p. [DM21,15]

Maxwell, A.K. et al., Anatomical Atlas for Nurses and Students, $4^{e}$ éd., Londres, Faber \& Faber, 1962, ill., 28 p.

Miller et Burack, Atlas of the Central Nervous Brain in Man, Baltimore (Md.), Williams \& Wilkins, 1968.

Monsei, H. (cf. Ferner), Pernkopf's Atlas of Topographical and Applied Human Anatomy (trad. de l'allemand), 2 vol., Philadelphie, Saunders, 1963-1964, ill., $356+431$ p. $[\$ 32.50+37.50]$

Olivier, G., les Nouveaux termes anatomiques, Paris, Vigot Frères, 1959, 148 p. [DM8]

Sobotta, J., Sobotta's Atlas of Human Anatomy, (trad. Uhlenhuth, E.), $7^{\text {e }}$ éd., 3 vol., Londres, Blackwell, 1957.

Spalteholz, W. et R. Spanner, Atlas of Human Anatomy, $16^{\mathrm{e}}$ éd., Londres, Butterworth, 1967, $916 \mathrm{p}$.

Woerdeman, H. W., Nomina anatomica parisiensia (1955) et B.N.A. (1895), Utrecht, 1957, $17+174 \mathrm{p}$.

Woerdeman, M.W., Atlas d'anatomie humaine, 2 vol., Paris, Presses Universitaires de France, 1968.

Wolff-Heidegger, G., Atlas of Systematic Human Anatomy, $2^{e}$ éd., 3 vol., Londres, Blackwell, 1962.

\subsubsection{Bactériologie}

Comité international de la nomenclature bactériologique, International Code of Nomenclature of Bacteria and Viruses, Ames, 1958, 186 p. 
Society of American Bacteriologists, Glossary of Bacteriology (en feuillets avec mise à jour), Genève, Biotechnical Publ., 1943.

Cowan, S.T., A Dictionary of Microbial Taxonomic Usage, Londres, Oliver \& Boyd, 1968, ill., $12+118$ p.

Hauduroy, P., Dictionnaire des bactéries pathogènes pour l'homme, les animaux et les plantes, $2^{\mathrm{e}}$ éd., Paris, Masson, 1953, $8+614+65 \mathrm{p}$.

Jacobs, M.A. et al., Dictionary of Microbiology, Princeton (N.J.), Van Nostrand, 1957, ill., 276 p.

Karel, L. et E.S. Roach, A Dictionary of Antibiosis, New York, Columbia Univ. Press, $1951,8+373$ p.

Wilner, B.I., Classification of the Major Groups of Human and other Animal Viruses, $3^{\mathrm{e}}$ éd., Minneapolis, $1966,91 \mathrm{p}$.

\subsubsection{Biochimie}

Glossaire de biochimie et de neurophysiologie cérébrales (français-anglais-néerlandais-espagnol), Bruxelles, UCB-div. pharmaceutique, 1972, 4 x 16 p. (hors commerce)

International Union of Biocemistry, Enzyme nomenclature, $2^{\mathrm{e}}$ éd., Amsterdam, Elsevier, 1966, $219 \mathrm{p}$.

Courtois, «La nomenclature des vitamines et des stéroïdes», Bull. Soc. Chim. Biol., 1956, vol. 38, p. 295-319.

Gajdos, «Dictionnaire enzymologique», Presse méd. (Paris), 1966, vol. 74, p. 319-322, 379-381.

Iseng, K.G., "Terminology of Seaweed Colloids», Science, 1945, vol. 101, $\mathrm{n}^{\circ} 2333$, p. 597-602.

Morton, H.A., International Encyclopedia of Food and Nutrition, vol. 19 : Fat-soluble Vitamins, Londres, Pergamon Press, 1969, 366 p.

Williams, R.J. et E.M. Lansford, Encyclopedia of Biochemistry, New York, Reinhold, $1967,17+876 \mathrm{p}$.

\subsubsection{Biologie}

Communautés européennes, Vocabulaires spécialisés : «Biologie moléculaire 》 (français-anglais-allemand-néerlandais-italien), Bulletin de la traduction, Bruxelles, Bureau Terminol, CEE, $1970 \mathrm{n}^{\circ}$ 40, p. 40-44.

The Living Universe, An Encyclopedia of the Biological Sciences, 5 vol., Londres, Nelson, 1964.

Fed. of Am. Societies for Experimental Biology, «Guide to Current Terminology in Biomedical Research », Federation's Proceedings, Bethesda (Md), 1965, p. $960-991$.

International Electrotechnical Commission, International Electrotechnical Vocabulary - Group 70 : Electrobiology (English-French), $2^{\mathrm{e}}$ éd., Genève, C.E.I., 1959.

Abercrombie, M. et al., A Dictionary of Biology, $5^{\mathrm{e}}$ éd., Harmondsworth, Penguin, 1966, ill., $254 \mathrm{p}$. 
Battro, A.M., Dictionnaire d'épistémologie génétique, Paris, 1966, 200 p.

Gray, P., The Dictionary of the Biological Sciences, New York, Reinhold Publ. Co., 1967, ill., $20+602$ p.

Gray, P., The Encyclopedia of Biological Sciences, New York, Reinhold Publ. Co., 1961, 1119 p.

Henderson, I.F., W.D. Henderson et J.W. Kenneth, Dictionary of Biological Terms, (anct. : Dictionary of Scientific Terms), $8^{e}$ éd., Édimbourg-Londres, Oliver \& Boyd, 1963, $15+640$ p.

Husson, R., Glossaire de biologie animale, Paris, Gauthiers-Villars, 1970, 300 p. Jaeger, E.C., A Source-Book of Biological Names and Terms, $3^{\text {e }}$ éd., Springfield, Thomas, 1966, ill., 360 p.

Sayers, N.F., A Biological Glossary, Londres, University Press, 1951, 168 p.

Shilling, C.W. et al., Atomic Energy Encyclopedia of the Life Sciences, Philadelphie, Saunders, 1964, ill., 414 p.

Swift, M., An Illustrated Dictionary for Students of Biology, Londres, Heinemann Medical, 1967, ill., $6+109$ p.

\subsubsection{Cancérologie}

International Union Against Cancer, Histological Nomenclature of Human Tumours - Nomenclature histologique des tumeurs humaines, 1958.

Hamperl, H., International Union Against Cancer, Illustrated Tumor Nomenclature, (English-Latin-French-German-Spanish-Russian), Berlin, Springer, 1965, ill., $32+299$ p.

Smalbraak, J., Trophoblastic Growths, (English-French-German-Spanish-Italian), Amsterdam, Elsevier, 1957, ill., $12+342$ p.

Zimmerman, Netsky et Davidoff, Atlas of Tumours of the Nervous System, New York, Lea \& Febiger, 1956, ill., 190 p.

Zülch, K.J. et A.L. Woolf, Classification of Brain Tumors, Berlin, Springer, 1965, ill., $10+218 \mathrm{p}$.

4.2.8. Cardiologie, électrocardiologie

U.S. National Heart Institute, $A$ Handbook of Heart Terms, U.S.N.H.I., 1964.

Nomenclature des actes d'électrocardiologie et d'électrothérapie, Paris, Librairies Techniques, 1960.

Deltour, Glossary of Technical Terms Used in Cardiovascular Pharmacology and Medical Therapeutics (French-English), mémoire, Bruxelles, Inst. sup. de traducteurs de l'État, 1968.

Edwards, An Atlas of Acquired Diseases of the Heart and Great Vessels, 3 vol., Philadelphie, Saunders, 1961, 1437 p.

Fattorusso, V. et O. Ritter, Atlas d'électrocardiographie, $7^{\mathrm{e}}$ éd., Paris, Masson, 1963.

Luisada, Cardiology : An Encyclopedia of the Cardiovascular System, 5 vol,, New York, Blakiston-McGraw, 1959-1963, + suppl,, ill., 5118 p. 
Sherman, Atlas of Congenital Heart Disease, New York, Lea \& Febiger 1963, ill., 303 p.

Trethewie, E.R., Atlas of ABC Electrocardiography (English-French-German), Londres, Blackwell, 1969, ill., 73 p.

4.2.9. Chirurgie, orthopédie, traumatologie

American Medical Association, A.M.A. Standard Nomenclature of Diseases and Operations, $5^{\mathrm{e}}$ éd., New York, Blakiston-McGraw, 1961, 964 p.

Beattie, E.J. et S.G. Economou, An Atlas of Advanced Surgical Techniques, Philadelphie, Saunders, 1968, ill., 422 p.

Clarke, M.K., Illustrated Guide to Theatre Instruments, Londres, Butterworth, 1966, ill., 86 p.

Cozen, Atlas of Orthopedic Surgery, New York, Lea \& Febiger, 1966, 732 p.

Harlow, F.W., An Atlas of Surgery, Londres, Blackwell, 1958.

Hershey et Calman, Atlas of Vascular Surgery, $2^{\mathrm{e}}$ éd., St. Louis (Minn.), Mosby, 1967, ill., 332 p.

Madden, Atlas of Techniques in Surgery, $2^{*}$ éd., 2 vol., New York, Appleton, 1964, ill., $1138 \mathrm{p}$.

Matthias, A.M., M. Penfold et S. Fry, Illustrated Guide for Theatre Nurses, Londres, Butterworth, 1962, ill., 94 p.

Méary, Code de classement des affections, interventions et références bibliographiques intéressant l'orthopédie et la traumatologie, Paris, 1966, 136 p.

Paton et Katzin, Atlas of Eye Surgery, $2^{\mathrm{e}}$ éd., New York, Blakiston-McGraw, 1962,492 p.

Poppen, J.L., An Atlas of Neurosurgical Techniques, Philadelphie, Saunders, 1960 , ill., 522 p.

Wilder, Atlas of General Surgery, $2^{\mathrm{e}}$ éd., St. Louis (Minn.), Mosby, 1964, 325 p.

Zollinger, R.H. et R.M., Atlas of Surgical Operations, $3^{\text {e }}$ éd., 2 vol., Londres, Macmillan, 1961-1967, $234+200$ p.

\subsubsection{Cytologie}

Darlington, D.C., Recent Advances in Cytology, Londres, J. \& A. Churchill, 1937, p. 572-83.

Heilmeyer, L. et H. Begeman, Atlas of Clinical Haematology and Cytology (English-French-German-Spanish), 2 vol., Berlin, Springer, 1955, ill., $411+$ $40 \mathrm{p}$.

4.2.11. Dentisterie, odontologie, stomatologie

Academy of Denture Prosthetics, Glossary of Prosthodontic Terms, St. Louis (La.), C.V. Mosby, 34 p. in : Journal of Prosthetic Dentistry, vol. 6, $\mathrm{n}^{\circ} 2$, 1956.

International Dental Federation, A Lexicon of English Dental Terms (EnglishFrench-German-Spanish-Italian), 1966.

Denton, G.B., The Vocabulary of Dentistry and Oral Science, Chicago, American Dental Association, 1958, 207 p. 
Filderman, M., «Le vocabulaire des chirurgiens-dentistes», Vie et langage (Paris), 1958, p. 547.

Fowler, J.E.H., The Heinemann Modern Dictionary for Dental Students and Nurses, Londres, Heinemann, 1962, ill., 32 p.

Moortgat, Odonto-stomatologie : syndromes à noms propres, 1966.

Roty, Termes employés en anatomie, pathologie et thérapeutique stomatologiques (anglais-français), mémoire, Bruxelles, Inst. sup. de traducteurs de l'État, 1970.

\subsubsection{Dermatologie}

Burckhardt et Epstein, Atlas and Manual of Dermatology and Venerealogy, $2^{\mathrm{e}}$ éd., Baltimore, Williams \& Wilkins, 1967, ill., 305 p.

Frieboes et al., Color Atlas of Dermatology, Philadelphie, Saunders, 1966, ill., $304 \mathrm{p}$.

de Graciansky, P., S. Boulle et M. Boulle, Atlas de dermatologie, Paris, Maloine, 1968.

Leider, M., A Dictionary of Dermatological Words, Terms and Phrases, New York, McGraw-Hill, 1968, $18+440 \mathrm{p}$.

Percival et al., An Atlas of Hispathology of the Skin, $2^{\mathrm{e}}$ éd., Baltimore, Williams \& Wilkins, 1962, $508 \mathrm{p}$.

Peters, J.A., Dictionary of Herpetology, New York, 1964, 392 p.

\subsubsection{Embryologie}

Henderson (voir Biologie).

\subsubsection{Endocrinologie}

Lisser, H. et R.F. Escamilla, Atlas of Clinical Endocrinology, $2^{\mathrm{e}}$ éd., St. Louis, Mosby, 1962, $489 \mathrm{p}$.

\subsubsection{5. Épidémiologie}

Biraud (voir Pathologie).

Gabaldon (voir Médecine tropicale).

Horn (voir Hygiène).

\subsubsection{Génétique}

Committee on Standardized Genetic Nomenclature for Mice, «Standardized Nomenclature for Inbred Strains of Mice», Cancer Research, vol. 24, 1964, p. 147-168.

Biass-Ducroux, F., Glossary of Genetics (English-French-German-Spanish-ItalianRussian), Amsterdam, Elsevier, 1970, $13+436$ p.

Clapper, R.B., Glossary of Genetics and other Biological Terms, New York, Vantage Press, 1961, $200 \mathrm{p}$.

Colin, E.C., Elements of Genetics, $3^{\text {e }}$ éd., New York, McGraw-Hill, 1956, p. $477-483$.

Goldschmidt, R.B. Understanding Heredity, New York, J. Wiley, 1952, p. 217221. 
Jepsen, G.L., Genetics, Paleonthology and Evolution, Princeton, Princeton University Press, 1949, p. 449-462.

King, R.C., A Dictionary of Genetics, New York, Oxford University Press, 1968, $291 \mathrm{p}$.

Knight, R.L., Dictionary of Genetics, Waltham (Mass.), Chronica Botanica Co., 1948, ill., 183 p.

Rieger, R., A. Michaelis et M.M. Green, A Glossary of Genetics and Cytogenetics, Londres, Blackwell, 1969, ill. 507 p.

\subsubsection{Génie sanitaire}

Pollution - Lexique français-anglais Conseil canadien des ministres des Ressources, Montréal, 1967.

British Standards Institution, Public Health Engineering Water Sanitation, Londres, B.S.I., 1968, BS 1000 (628), 27 p.

World Health Organization, A Glossary of Water and Sewage Terms used in Sanitary Engineering (English-French), Genève, WHO-OMS, 1956, 74 + 98 p.

Besselievre, F.R., Industrial Waste Treatment, New York, McGraw-Hill, 1952, p. 353-365.

Isaac, P.S.G., A Glossary of Sanitary Engineering Terms, Newcastle-upon-Tyne, Univ. of Durham, 1955, $72 \mathrm{p}$.

Kaupert, W., Dictionary of Waste Disposal and Public Cleansing (English-FrenchGerman), Amsterdam, Elsevier, 1966, $7+224$ p.

Meyer, A.F. et al., Trinkwasser und Abwasser in Stichwörten, (français-anglaisallemand-italien), Berlin, Springer, 1949, $487 \mathrm{p}$.

Sommer, W., Methods of Air Deodorization, Amsterdam, Elsevier, 1963, p. 375376.

4.2.18. Gynécologie, obstétrique

Breen et Jaffurs, An Atlas of Gynecologic and Obstetrical Pathology, Davis, 1968, 200 dias sur feuillets mobiles.

Burnett, C.F.W., The Faber Pocket Dictionary of Midwifery, Londres, Faber, 1966, ill., $11+334$ p.

Carter, G.B. et al., A Dictionary of Midwifery and Public Health, $2^{\mathrm{e}}$ éd., Londres, Faber \& Faber, 1963, ill.

Janovski et al., Atlas of Gynecological and Obstetric Diagnostic Histopathology, New York, Blakiston-McGraw, 1967, ill., 620 p.

Janovski et al., Color Atlas of Gross Gynecologic and Obstetric Pathology, New York, Blakiston-McGraw, 1968.

Louros, N.C., Obstétrique et gynécologie (Glossaria interpretum-G8) Amsterdam, Elsevier, 1964, $7+444$ p.

Postoloff et Nichols, Atlas of Gynecologic Pathology, Baltimore, Williams \& Wilkins, 1952,100 dias $+86 \mathrm{p}$. 


\subsubsection{Hématologie}

Block, Atlas of Hematology, New York, Lea \& Febiger, 1968.

Daland, A Color Atlas of Morphologic Hematology, $2^{\mathrm{e}}$ éd., Cambridge, Harvard Univ. Press, 1959, 72 p.

Dunsford, I. et C.C. Bowley, Techniques in Blood Grouping, Édimbourg, Oliver \& Boyd, 1955, p. 213-232.

McDonald, G.A. et al., Atlas of Hematology, Baltimore, Williams \& Wilkins, 1965, ill., $172 \mathrm{p}$.

Heilmeyer, L. (voir Cytologie).

Romei Braconi L., Hematology (Glossaria interpretum - G9), Amsterdam, Elsevier, $1964,7+306 \mathrm{p}$.

4.2.20. Histologie

Curran, R.C., A Color of Histopathology, New York, Oxford University Press, 1966 , ill., $100 \mathrm{p}$.

Di Fiore, M.S.H., Atlas of Human Histology, $3^{\mathrm{e}}$ éd., Londres, Blackwell, 1967, $243 \mathrm{p}$.

Freeman, W.H. et B. Bracegirdle, An Atlas of Histology, Londres, Blackwell, 1965.

Henderson (voir Biologie).

Lovasy (voir Anatomie).

Iliero, S.J. et al., Atlas of Histology, Philadelphie, Lippincott, 1965.

Stiles, K.A., Handbook of Microscopic Characteristics of Tissues and Organs, Philadelphie, Blakiston, 1948, p. 203-209.

Valdes-Dapena, An Atlas of Fetal and Neonatal Histology, Philadelphie, Lippincott, 1957, ill., $200 \mathrm{p}$.

von Herrath, E., Atlas of Histology and Normal Microscopic Anatomy of Man, trad. de l'allemand, $2^{2}$ éd., New York, Hafner, 1966.

\subsubsection{Homéopathie}

Maury, F.A., Dictionnaire familial d'homéopathie, Paris, 1965, 148 p.

Pommier, L., Dictionnaire homéopathique d'urgence, $9^{\text {e }}$ éd., Paris, Chez l'auteur, $1965,796 \mathrm{p}$.

4.2.22. Hygiène (cf. aussi : génie sanitaire)

Encyclopedia of Instrumentation for Industrial Hygiene, Ann Arbor, Univ. of Michigan, 1956.

Carter (voir Gynécologie).

Chenoweth, L.B. et T.K. Selkirk, School Health Problems, $4^{\mathrm{e}}$ éd., Croft, Appleton Century, 1953, p. 429-440.

\subsubsection{Immunologie}

Herbert, W.J. et al., A Dictionary of Immunology, Oxford, Blackwell, 1971, 195 p.

4.2.24. Instrumentation, techniques, laboratoire

International Committee on Laboratory Animals, Recommended Definitions for 
Current Terms Employed in the Care, Maintenance and. Use of Laboratory Animals, 1963.

International Organization for Standardization, Conical Fittings for Syringes, Needles and Other Medical Equipment - Assemblages côniques des seringues, des aiguilles et de certains appareils à usage médical, Genève, 1967, ISO R 594.

International Organization for Standardization, Hypodermic Needles - Aiguilles hypodermiques, Genève, 1967, ISO R 596.

International Organization for Standardization, Syringes for Medical Use - Seringues à usage médical, Genève, 1966, ISO R 740.

International Organization for Standardization, Transfusion Equipment for Medical Use - Matériel de transfusion à usage médical, Genève, 1966, ISO R 1221.

Clark, G.L., Encyclopedia of Microscopy, New York, Reinhold, 1961, ill., 708 p.

Mark et Zimmer, Atlas of Clinical Laboratory Procedures - vol. I : Clinical Chemistry, New York, Blakiston-McGraw, 1967, ill., 188 p.

Syllaba, J., Méthodes d'examen physiques en médecine interne (palpation, percussion, auscultation, diagnostic instrumental), $2^{\mathrm{e}}$ éd., Prague, SZdN, 1958, ill., $437 \mathrm{p}$.

4.2.25. Médecine aéronautique et spatiale

Advisory Group for Aeronautical Research and Development (A.G.A.R.D.), A Glossary of Terms Commonly Used in Aviation Medecine (English-French, French-English), Londres, NATO, 1959, 30 p.

4.2.26. Médecine légale

Camps, F.E. et W.B. Purchase, Practical Forensic Medecine : A Concise Encyclopedia, Londres, Blackwell, 1956.

Levitt, W.M., Short Encyclopedia of Medicine for Lawyers, Londres, Butterworth, 1966, ill., $482 \mathrm{p}$.

Porot (voir Psychiatrie).

Schmidt, J.W., Police Medical Dictionary, Springfield, Thomas, 1968, $7+246$ p.

Watanabe, T., Atlas of Legal Medecine, Londres, Blackwell, 1969, ill., 187 p.

\subsubsection{Médecine militaire}

Vade-Mecum medio-chirurgical (français-anglais), Dinard, Plumon, 1940, 350 p.

\subsubsection{Médecine nucléaire}

Etter, L.E. (voir Radiologie).

4.2.29. Médecine du travail

Cuvier, G.A., U. Cavigneaux et R. et G. Vallet, Index pratique des maladies professionnelles : commerce, industrie, agriculture, Paris, Les Éditions sociales françaises, $1967,164 \mathrm{p}$.

4.2.30. Médecine sportive

Encyclopedia of Sports Medicine, Londres, Colliers-Macmillan, 1971, $47+$ $1707 \mathrm{p}$. 


\subsubsection{Médecine tropicale}

Baghio'oh, Dictionnaire de médecine tropicale, Paris, Maloine, 1960, 56 p.

Covell, Russel et Swellengrebel, Malaria Terminology, 1953.

Gabaldion, A. et al., World Health Organization, Terminology of Malaria and of Malaria Eradication, Genève, WHO-OMS, 1963, 127 p.

Vaucel, M., E. Rouhaud et H. Galliard, Terminologie du paludisme, Genève, WHO-OMS, 1954, 95 p. (Texte parallèle à celui de Malaria Terminology de Covell et al.)

\subsubsection{Médecine vétérinaire}

Allied Control Authority of Germany, Dictionary of Common Infectious Diseases of Domestic Animals (Latin-English-French-German-Russian), USC/USA, 1946, (46) $9,5 \mathrm{p}$.

Merck, Sharpe and Dohme Research Laboratories, The Merck Veterinary Manual, Handbook of Diagnosis and Therapy, $3^{\mathrm{e}}$ éd., Rahway (N.J.), Merck \& Co., $1967,1680 \mathrm{p}$.

Brion, A., Formulaire vétérinaire de pharmacologie, de thérapeutique et d'hygiène, $12^{e}$ éd., Paris, Vigot Frères, 1966, 703 p.

Husson (voir Biologie).

Merino-Rodriguez, M., Lexicon of Parasites and Diseases in Livestock (LatinEnglish-French-German-Italian), Amsterdam, Elsevier, 1964, $8+125$ p.

Miller, W. C., Encyclopedia of Animal Care (anct.: Black's Veterinary Dictionary), $7^{\mathrm{e}}$ éd., Baltimore, Williams \& Wilkins, 1964, ill., $8+1017$ p.

Miller, W.C. et G.P. West, Black's Veterinary Dictionary, $9^{\mathrm{e}}$ éd., Londres, Blackwell, 1970, ill., $8+1013 \mathrm{p}$.

Schulz, H.E., Vocabularium veterinarium Polyglotte (latin-français-anglais-allemand-espagnol-italien-russe), 2 vol. classeurs, Halle (Westf.), Deutsches Nomenklatur-Arch., 1961, 292 p.

Slack et Nineham (voir Pharmacie).

Villemin, M., Dictionnaire des termes vétérinaires et zootechniques, Paris, Vigot Frères, $1963,360 \mathrm{p}$.

\subsubsection{Mycologie}

Moss, E.S. et A.L. Mc Quown, Altas of Medical Mycology, $2^{\mathrm{e}}$ éd., Baltimore, Williams \& Wilkins, 1960, p. 226-232.

Snell, W.W. et E.A. Dick, A Glossary of Mycology, Cambridge (Mass.), 1957, ill., $171 \mathrm{p}$.

4.2.34. Neurologie, électroencéphalographie

International Federation of Societies for Electroencephalography and Clinical Neurophysiology, "Preliminary Proposal for an EEG Terminology », Electroencephalography Clinical Neurophysiology, 1961, vol. 13, p. 646-650.

Blackwood et al., Atlas of Neuropathology, $2^{\mathrm{e}}$ éd., Baltimore, Williams \& Wilkins, 1965, ill., $246 \mathrm{p}$.

Burkhardt (voir Physiologic). 
Gibbs et Gibbs, Atlas of Encephalography, 3 vol., Reading (Mass.), Addison-

Wesley, 1950, 1952, 1964, ill., $324+426+540$ p. [\$165.]

McCormick, J.B., Atlas and Demonstration Technique of the Central Nervous System, Springfield (II1.), 1961, 116 p. [\$11.25]

Poppen (voir Chirurgie).

Poser (voir Pharmacie).

Spencer (voir Pathologie).

Spillane, An Atlas of Clinical Neurology, New York, Oxford University Press, 1968.

4.2.35. Nursing, soins infirmiers

Comité d'étude des termes de médecine, Glossaire des termes médico-hospitaliers, français-anglais, Montréal, Laboratoires Ayerst, 1966.

Cape, B.F., Baillière's Nurse's Dictionary, $17^{\mathrm{e}}$ éd., Londres, Baillière, Tindall \& Cassell, 1968, ill., 544 p.

Matthias, A.M., M. Penfold et S. Fry, (voir Chirurgie).

Monceaux, J.P., Lexique des termes médicaux à l'usage des infirmières, Paris, Lamarre-Poinat, 1965,79 p.

Morten, H. et P.J. Cunningham, The Nurse's Dictionary, 26éd., Londres, Faber $\&$ Faber, 1966, ill., 430 p.

Morton, L.T. et J.J. Abraham, The Heinemann Modern Dictionary for Nurses, Londres, Heinemann, 1961, ill., 320 p.

Oakes, L. et T.B. Davie, Dictionary for Nurses, $12^{\circ}$ éd., Édimbourg, E. \& S'. Livingstone, $1966,560 \mathrm{p}$.

Olson-Dorland, A Reference Handbook and Dictionary of Nursing, Philadelphia, Saunders, 1968, ill., 1224 p. [\$7.00]

Pearce (voir Médecine générale).

Petry, L. et al., The Encyclopedia of Nursing, Philadelphia, Saunders, 1952, ill., $1011 \mathrm{p}$.

Price, A.L., The American Nurse's Dictionary, Philadalphia, Saunders, 1949, $656 \mathrm{p}$.

Schmidt, J.E., Practical Nurses' Medical Dictionary, Springfield, Thomas, 1968, 300 p. [\$6.95]

\subsubsection{Nutrition, diététique}

Food and Agriculture Organization, Selected Terms in Nutrition and Home Economics (English-French-Spanish), Rome, FAO, 1968.

Bender, A.E., Dictionary of Nutrition and Food Technology, $3^{\text {e }}$ éd., New York, Academic Press, 1968, 238 p. [\$6.00]

Cooper, L.F. et al., Nutrition in Health and Disease, $12^{*}$ éd., Philadelphie, Lippincott, 1953, p. 747-754.

Ellis, R., Dictionary of Dietetics, Londres, Owen, 1956, 152 p.

Lassabiière, Lesne et Tanon, Encyclopédie de l'alimentation scientifique, médicale, hygiénique et gastronomique, Paris, Maloine, 1955. 
Sinclair, H.M. et al., International Encyclopedia of Food and Nutrition, Londres, Pergamon Press.

\subsubsection{Ophtalmologie}

Alvaro, M.E. et al., Lexicon Ophtalmologicum (latin-français-anglais-allemandespagnol-italien), Bâle, Karger, 1959, $6+217$ p.

Barsky, Color Atlas of the Pathology of the Eye, New York, Blakiston-McGraw, 1966, ill., 239 p. [\$30.00]

Donaldson, Atlas of External Diseases of the Eye, 2 vol., St. Louis, Mosby, 1966-1968, ill., 400 p. [\$70.00]

Hardy, W.E. et al., Dictionary for Opticians and Optometrists, Londres, Halton Press, 1952, $166 \mathrm{p}$.

Perkins et Hansell, An Atlas of Diseases of the Eye, Little, Brown \& Co., 1957, ill., 91 p. [\$13.50]

Schapero, M., D. Cline et H.W. Hofstetter, Dictionary of Visual Science, $2^{\mathrm{e}}$ éd., Philadelphie, Chilton, 1968, $22+804$ p. [\$24.95]

Schappert-Kimmijser, J. et al., Coding Systems for Disorders of the Eye, Bâle, Karger, 1968, $4+206$ p.

Thiel, R., Atlas of Diseases of the Eye, 2 vol., trad. de l'allemand, Amsterdam, Elsevier, 1963, ill., 16/350 + 12/323 p.

\subsubsection{Orthopédie}

Quinet, Glossary of Orthopaedics (English-French), mémoire, Bruxelles, Inst. sup. de traducteurs de l'État, 1968.

\subsubsection{Oto-rhino-laryngologie}

Arslan, M. et A. Bosatra, Otorhinolaryngology, Amsterdam, Elsevier, sous presse.

Becker, W. et al., Atlas des maladies oto-rhino-laryngologiques, traduction de l'allemand, Paris, Maloine, 1969, ill., 324 p. Existe aussi en version anglaise.

4.2.40. Parasitologie

Maer, J.G. et al., Tabulae parasitorum intestinorum, Stuttgart, Thierme, 1955, ill., 55 p. [\$5.50]

Jeffrey et Leach, Atlas of Medical Helminthology and Protozoology, Baltimore, Williams \& Wilkins, 1966, ill., 150 p. [\$15.75]

Spencer, F.M. et L.S. Monroe, The Color Atlas of Intestinal Parasites, Springfield, Thomas, 1968, ill., 160 p. [\$9.50]

\subsubsection{Pathologie}

American Medical Association, A.M.A. Standard Nomenclature of Diseases and Operations, $5^{\mathrm{e}}$ éd., New York, Blakiston-McGraw, 1961, 964 p. [\$12:50]

Laboratoires Midy, Petit dictionnaire des maladies rares, Paris, 1955, $36 \mathrm{p}$.

National Naval Medical Center, Color Atlas of Pathology, 3 vol., Philadelphie, Lippincott, 1950-1954-1963, ill., $546+450+348$ p. [\$75.00]

Theraplik, Glossaire de rhumathologie, Paris, Rhone-Poulenc, 1970, 102 p. (Hors commerce.) 
World Health Organization, International Statistical Classification of Diseases, Injuries and Causes of Death, 2 vol., Genève, WHO-OMS, 1957, $41+393$ p., $26+540$ p.

Biraud, Y., «Lexique polyglotte des maladies contagieuses», League of Nations Health Organisation Quaterly Bulletin, vol. 10, $\mathrm{n}^{\circ} 3,1943-1944$, p. 201-256.

Breen et Jaffurs (voir Gynécologie).

Colby, Kerr et Robinson, Color Atlas of Oral Pathology, $2^{\mathrm{e}}$ éd., Philadelphie, Lippincott, 1961, 201 p. [\$15.25]

Durham, Encyclopedia of Medical Syndromes.

Gomez, J., A Dictionary of Symptoms, Londres, Paladin, 1970, 490 p.

Gomez, J. (trad. P. Alexander), Dictionnaire encyclopédique des symptômes, Paris, Planète, 1970,608 p.

Hackett, C.J. et al., Nomenclature internationale des lésions planiques, Paris, 1958, ill., $104 \mathrm{p}$.

Israels, An Atlas of Bone-Marrow Pathology, Grune \& Stratton, 1967, ill., 96 p. [\$8.50]

Jablonski, S., Illustrated Dictionary of Eponymic Syndromes and Diseases, Philadelphie, Saunders, 1969, ill., 800 p.

Janovski (voir Gynécologie).

Kaiser-Meinhardt (voir Oto-rhino-laryngologie).

Plunkett, R., A.C. Hayden et al., Standard Nomenclature of Diseases and Operations, $4^{e}$ éd., Philadelphie, Blakiston, 1952, ill., $16+1034$ p.

Postoloff et Nichols (voir Gynécologie).

Robbins, S.D., Dictionary of Speech Pathology and Therapy, Cambridge, ScienceArt Publications, 1951, 112 p.

Ruhl et Sokoloff, $A$ Theasaurus of Rhumatology, 1965.

Sandritter, Color Atlas and Textbook of Tissue and Cellular Pathology, New York, Year Book, 1967, ill., 298 p. [\$21.00]

Spencer, H., A Glossary of Scientific Terms in the Field of Cerebral Palsy, New York, Columbia Univ. Press, 1956, 26 p.

Than, Lexicon of the Diseases of Digestion and Nutrition (French-EnglishSpanish), mémoire, Bruxelles, Inst. sup. de traducteurs de l'État, 1966.

Young, C.G., Medical Specialty Terminology - Vol. I : Pathology, Londres, Kimpton, 1971, 464 p.

4.2.42. Pédiatrie

Lafon (voir Psychiatrie).

Moor (voir Psychiatrie).

\subsubsection{Physiologie}

Blacque-Bélair, A., B.M. de Fossey et M. Fourestier, Dictionnaire des constantes biologiques et physiques; applications cliniques et explorations paracliniques (anct. : Dictionnaire des constantes biologiques), $4^{\mathrm{e}}$ éd., rev. et ref., Paris, 1965, ill., 960 p. 
Fourestier, M. et B.M. de Fossey, Dictionnaire des constantes biologiques et leurs interprétations, $3^{\mathrm{e}}$ éd., Paris, 1951 , ill., $450 \mathrm{p}$.

Henderson (voir Biologie).

Hiebert (voir Abréviations).

\subsubsection{Psychiatrie}

Chamber's Dictionary of Psychiatry, Londres, Chambers, 1967.

American Psychiatric Association, A Psychiatric Glossary, Washington, 1964, 80 p. [\$2.50]

Secrétariat d'État, Centre de terminologique (Canada), Terminologie de la psychiatrie (anglais-français), Ottawa, 1955, BT-30, polycopie, $43 \mathrm{p}$.

Brussel, J.A. et G. Cantzlaar, Dictionary of Psychiatry, New York, Barnes \& Noble, 1966. $[\$ 5.50]$

Des Vignes Rouges, J., Dictionnaire de la guérison, Paris, Oliven, 1947, 256 p.

Dujardin, J.L., Drug-Addiction in French and English, thèse, Anvers, 1967, 149 p.

Eidelberg, L., Encyclopedia of Psycho-Analysis, New York, Free Press, 1968, $73+571$ p. [\$27.50]

English, H.B. et A.C. English, A Comprehensive Dictionary of Psychological and Psychoanalytical Terms, New York, Longmans, Green \& Co., 1959, 608 p. [\$10.75]

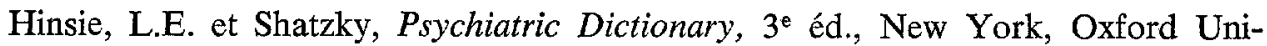
versity Press, 1960,798 p. [\$17.50]

Hinsie, L.E. et P.J. Campbell, Psychiatric Dictionary, $4^{\mathrm{e}}$ éd., Riverside (N.J.), Behavioral Science Book Service, 1970. [\$19.50]

Lafon, R., Vocabulaire de psychopédagogie et de psychiatrie de l'enfant, Paris, $1963,20+604 \mathrm{p}$.

Laplanche, J. et J.B. Pontalis, Vocabulaire de la psychoanalyse (français-anglaisallemand-espagnol-italien-portugais), Paris, Presses Universitaires de France, $1967,540 \mathrm{p}$.

Marchais, P., Glossaire de psychiatrie, Paris, Masson, 1970, 258 p.

Moor, L., Glossaire de psychiatrie, de psychologie pathologique et de neuropsychiatrie infantile, Paris, Masson, 1966, $196 \mathrm{p}$.

Moor, L., Lexique des termes usuels en psychiatrie, neuropsychiatrie infantile et psychologie pathologique (français-anglais-allemand), $2^{e}$ éd., Paris, Expansion scientifique française, $1969,224 \mathrm{p}$.

Neugebauer, J., C. Weil, et World Psychiatric Association, Common Psychiatric Terms in 4 Languages (English, French, German, Spanish), Bâle, Sandoz, 1971, 149 p. (Hors commerce.)

Nyswander, M., The Drug Addict as a Patient, New York, Grune \& Stratton, 1956, p. 171-172.

Porot, A., Manuel alphabétique de psychiatrie clinique, thérapeutique et médicolégale, $2^{\mathrm{e}}$ éd., Paris, Presses Universitaires de France, 1960, $8+437$ p.

Poser (voir Pharmacie). 
Rosanoff, A.J., "Official Classification of Mental Disorders Followed by Definitions », Manual of Psychiatry and Mental Hygiene, New York, Wiley \& Sons, 1947, p. 967-985.

Rycroft, Ch., A Critical Dictionary of Psycho-Analysis, Londres, Nelson, 1969.

\subsubsection{Radiologie}

British Standards Institution, Glossary of Terms Used in Radiology (EnglishFrench-German), Londres, B.S.I., 1955, B.S. 2597, ill., 82 p.

International Electrotechnical Commission, International Electrotechnical Vocabulary-Group 65: Radiology and Radiological Physics (English-French, French-English), $2^{\circ}$ éd., Genève, C.E.I., 1964.

Clark, G.L., Encyclopedia of X-Rays and Gamma-Rays, New York, 1963, 1178 p. [\$37.50]

Davies, P.M., Medical Terminology of Radiographers, $2^{\mathrm{e}}$ éd., Londres, Heinemann, $1967,274 \mathrm{p}$.

Etter, L.E., Glossary of Words and Phrases Used in Radiology, Nuclear Medecine and Ultrasound, $2^{\mathrm{e}}$ éd., Springfield, Thomas, 1970, ill., 356 p. [\$23.50]

Hayden, H.P., Vocabulary to Roentgenographic Report, St. Paul (Minn.), Bruce, 1968,148 p. [\$3.50]

Rose, H.M., Medical Radiographic Terminology, Ann Arbor (Mich.), Edwards, $1955,108 \mathrm{p}$.

\subsubsection{Sexologie}

Dickinson, Atlas of Human Sex Anatomy, $2^{\mathbf{e}}$ éd., Baltimore, Williams \& Wilkins, 1949,346 p. [\$10.00]

Ellis, A., The Encyclopedia of Sexual Behavior, $2^{\mathrm{e}}$ éd., New York, Hawthorn, 1967,1072 p. [\$14.95]

Lo Duca, J.M. et al., Dictionnaire de sexologie, Paris, Pauvert, 1962, ill., 600 p.

Lo Duca, J.M., Nouveau dictionnaire de sexologie (supplément), Paris, Pauvert, 19667, ill., 432 p.

Reissner, A. et C. Wade, Dictionary of Sexual Terms, Bridgeport (Conn.), Assoc. Booksellers, 19667, ill., 158 p. [\$3.00]

\subsubsection{Thérapeutique}

Encyclopédie médico-chirurgicale - Kinésithérapie, 3 vol., Paris, Éd. Techniques, 1969 , ill., $960 \mathrm{p}$.

International Encyclopedia of Pharmacology and Therapeutics, Oxford, Pergamon Press, plus de 100 volumes.

Secrétariat d'État, Service de terminologie (Canada), la Réadaptation des invalides - Rehabilitation of Disabled Persons, Ottawa, 1955, BT-28, polycopie, $28 \mathrm{p}$.

Allison, R.S. et T.H. Crozier, Whitlas's Dictionary of Medical Treatment, $9^{\mathbf{e}}$ éd., Londres, Baillière, Tindall \& Cassell, 1957.

Des Vignes Rouges (voir Psychiatrie). 
Drouin, $\mathrm{H}$. et $\mathrm{C}$. Giraudet, Lexique des thérapeutiques quotidiennes, Paris, Soc. nouv. de Publ. Médicales, 1966, 434 p.

Kamenetz, H.L., Physiatric Dictionary - Glossary of Physical Medicine and Rehabilitation, Springfield, Thomas, 1965, 181 p. [\$7.00]

Perlemuter, L. et al., Dictionnaire pratique de thérapeutique médicale, Paris, Masson, 1971, $1230 \mathrm{p}$.

Porot (voir Psychiatrie).

Win, R.B., Dictionary of Hypnosis, Londres, Vision, 1965.

\subsubsection{Urologie}

Alken, C.E. et al., Encyclopedia of Urology - Handbuch der Urologie - Encyclopédie d'urologie, Berlin, Springer, 1965. (En cours de publication, nombreux vol. ill., le vol. environ 500-600 p.)

Reuter, H.J., Atlas der urologischen Endoskopie, 2e éd., Stuttgart, Thieme, 1963, ill., $10+117$ p. (Existe en versions française, anglaise, espagnole.)

4.3. PHARMACIE, PHARMACOLOGIE

Codex medicamentarius Gallicus - Pharmacopée française, $8^{e}$ éd., Paris, Ordre national des pharmaciens, 1965-1968, + suppl., $1902+159$ p.

International Encyclopedia of Pharmacology and Therapeutics, Oxford, Pergamon Press. (Plus de 100 vol. en cours de publication.)

Modern Drug Encyclopedia and Therapeutic Index, $9^{\mathrm{e}}$ éd., New York, Drug Publishing, 1963, 1649 p. [\$18.50]

Pharmacopée belge, 3 vol., $5^{\circledR}$ éd., Bruxelles, Ministère de la Santé publique, 1962.

The Extra Pharmacopoeia, 2 vol, $25^{\mathrm{e}}$ éd, Martindale, 1967.

The Pharmacopoeia of the United States of America, $17^{e}$ éd., New York, The US Pharmacopeal Convention, 1965, 1222 p. [\$12.50]

American Medical Association, New and Non Official Drugs, Philadelphie, Lippincott, 1966,592 p. [\$5.00]

Comité d'étude des termes de médecine, Glossaire des termes pharmaceutiques anglais-français, français-anglais, Montréal, Laboratoires Ayerst, 1969, 55 p.

Conseil de l'Europe, Pharmacopée européenne, Strasbourg, 1969, vol. 1, 430 p.

Conseil de l'Europe, Glossary in 5 Languages of the Principal Pharmaceutical Terms (French-English-German-Deutch-Portuguese), Strasbourg, 1963, 145 p. $[\$ 4.00]$

General Medical Council, British Pharmacopoeia, Londres, The Pharmaceutical Press, 1968, 1423 p.

Institute for Advancement of Medical Communication, Index-Handbook of Cardiovascular Agents, 2 vol., New York, Blakiston-McGraw, 1963-1965, 2067 + 1235 p. [\$65.00]

Organisation mondiale de la santé, Dénominations communes internationales des substances pharmaceutiques, Genève, OMS, Service de terminologie, 1970.

Organisation mondiale de la santé, «Empfohlene internationale freie Bezeichnungen für Arzneimittel (latin-français-anglais) », Osterreichisches Patentblatt, 1959-1960, vol. $56, \mathrm{n}^{\circ} 5$, p. $88-90+103-114$; vol. 67 , no 3 , p. 57-69. 
Organisation mondiale de la santé, Multilingual List of Narcotic Drugs under International Control, Genève, WHO-OMS, 1958, $8+102 \mathrm{p}$.

Organisation mondiale de la santé, Pharmacopoeia internationalis, 2 vol. + suppl., Genève, WHO-OMS, 1951-1960, 20/438 + 24/384 + 24/230 p.

Pharmaceutical Society of Great Britain, British Pharmaceutical Codex, Londres, The Pharmaceutical Press, 1968.

Société suisse de pharmacie, Subsidia pharmaceutica - Index nominum, 19631965.

US Adopted Names Council, United States Adopted Names : 1961-1965 Cumulative List, 1966.

Bovet, D. et al., International Encyclopedia of Pharmacology and Therapeutics, Londres, Pergamon Press.

Chaplet, Dictionnaire des produits chimiques commerciaux et des drogues industrielles, $5^{\mathrm{e}}$ éd., Paris, Dunod, 1957, ill. $8+576 \mathrm{p}$.

Chopra, R.N., S.L. Nayar et I.C. Chopra (Council of Scientific and Industrial Research, New Delhi), Glossary of Indian Medicinal Plants, Oxford, Pergamon Press, $1956,20+330 \mathrm{p}$.

Clairville, A.L., le Livre du praticien - Dictionnaire des nouveautés thérapeutiques - Dictionnaire pharmaceutique systématique - Dictionnaire alphabétique des spécialités pharmaceutiques, $8^{e}$ éd., Paris, S.I.P.U.C.O., 1955, $576 \mathrm{p}$.

Clarke, J.H., A Dictionary of Practical Materia Medica, 3 vol., Londres, Homoeopathic Publ. Co., 1955.

Donadieu, Y. et E. Albrecht, Thérapeutique psychotrope, classification et nomenclature des médications chimiothérapiques, $2^{e}$ éd., Paris, Maloine, 1966, 238 p.

Drouin, H., Lexique des spécialités pharmaceutiques, Paris, Legrand, 1957, 574 p.

Falconer, M.W. et al., Current Drug Handbook 1968-1970, Philadelphie, Saunders, 1968, 198 p. [\$3.75]

Fatturusso, V. et O. Ritter, Dictionnaire de pharmacologie clinique, (à l'usage du médecin, du pharmacien et de l'étudiant), Paris, Masson, 1967, 6 + $830 \mathrm{p}$.

Fleck, H.R., Synthetic Drugs - A Handbook for Chemists, Physicians and Pharmacists, Londres, Cleaver-Hume Press, 1955, 380 p.

Fournier, le Livre des plantes médicinales et vénéneuses de France (latin-françaisanglais-allemand-italien), 3 vol., Paris, Lechevalier, 1947-1948, $448+504$ $+636 \mathrm{p}$.

Heinige, A. et al., Index internationalis pharmaceuticus (latin-français-anglaisallemand-russe), Prag, Artia, 1968, $17+867 \mathrm{p}$.

Heraud, Dictionnaire des plantes médicinales, Paris, Baillière, 1949, 756 p.

Hocking, G.M., A Dictionary of Terms in Pharmacognosy and other Divisions of Economic Botany, Springfield, Thomas, 1965, 284 p. [\$8.00] 
Hopkins, S.J., Principal Drugs : An Alphabetical Guide, $3^{\mathrm{e}}$ éd., Londres, Faber $\&$ Faber, 1969.

Hua, H., Medical Drugs Terminology (Latin-English-French-German), $7^{\mathrm{e}}$ éd., Shanghai, Commercial Press, 1947, 50 p.

Jablonski, S., Russian Drug Index, $2^{\mathrm{e}}$ éd., Bethesda, 1967, 384 p.

Krasnoff, D.G., Fachworterbuch der Pharmazie (allemand-français-anglais-néerlandais-espagnol-italien), Amsterdam, Elsevier.

Leclerc, J. et al., Formulaire pharmaceutique, avec un lexique médico-pharmaceutique, Paris, Vigot, 1965, 2080 p.

Marler, E.E.J. et al., Pharmacological and Chemical Synonyms, $4^{\mathrm{e}}$ éd., Amsterdam, $1967,7+349 \mathrm{p}$.

Modell, Drugs in Current Use, New York, Springer, 1968, 160 p. [\$2.75, éd. annuelle.]

Pöldinger, W. et P. Schmidlin, Index psychopharmacorum, français-anglais-allemand, $2^{\circ}$ éd., Berne, Huber, 1966, 88 p.

Poser, C.M. et V. Osborn, International Dictionary of Drugs Used in Neurology and Psychiatry, Springfield, Thomas, 1962, $11+$ p. [\$7.50]

Rousset, H., Dictionnaire des produits chimiques commerciaux et des drogues industrielles, $5^{\mathrm{e}}$ éd., Paris, Dunod, 1957, 564 p.

Schmidt, J.E., Narcotics : Lingo and Lore, Springfield, Thomas, 1959, 216 p.

Slack, R. et A.W. Nineham, Medical and Veterinary Chemicals (vol. II : A Tabulated Classification of Drug Categories), Londres, Pergamon Press, 1968, $214 \mathrm{p}$.

Sliosberg, A., Dictionary of Pharmaceutical Science and Techniques, Amsterdam, Elsevier, 1968, vol. I : $10+686$ p., vol II : à paraître.

Stecher, P.G. et al., The Merck Index - An Encyclopedia of Chemicals and Drugs, $8^{e}$ éd., Rahway (N.Y.), Merck Labs, 1968, 1641 p.

Steinmetz, E.F., Drug Guide For Importers, Exporters, Dealers, etc., of Botanical Drugs and Spices Throughout the World (English-French-German), Amsterdam, Steinmetz, 1959, $134+134$ p.

Steinmetz, E.F., Materia medica vegetabilis (latin-français-anglais-allemand-néerlandais), 3 vol., Amsterdam, Steinmetz, 1954, 800 p.

Tompkins, G.P., Medicinal Products, U.S. Equivalents and Alternatives : Alkaloids, Biologicals, Chemicals, Glandular Products, Pharmaceutical Specialties, Vitamins (Spanish-Portuguese-English), Washington, US Government Printing Office, $1945,107 \mathrm{p}$.

Vieillefosse, O.R., Dictionnaire de pharmacologie animale, Paris, Maloine, 228 p.

Vidal, L., Dictionnaire Vidal 1966, $9^{\mathrm{e}}$ éd., Paris, Office de vulgarisation pharmaceutique, 1965, $2564 \mathrm{p}$.

Wilson, C.O. et T.E. Jones, American Drug Index, Philadelphia, 1967, 785 p. [\$7.50] 